\title{
Realidade Aumentada Aplicada ao Processo de Reabilitação Física de Membro Superior
}

\author{
Jone Follmann, Gabriel Brinhol, Patrick Tarouco, Julio Saraçol, Érico Amaral \\ Engenharia de Computação - Universidade Federal do Pampa (UNIPAMPA) \\ Bagé - RS - Brazil
}

\begin{abstract}
This work proposes a solution to support the process of the rehabilitation of patients who have suffered traumatic or surgical amputation of upper limbs. Considering this scenario, it is expected to provide a training tool that uses Augmented Reality (AR) Technology to assists the patients into the rehabilitation process. It is expected that when using this resource, the patients can be easily adapted to a physical prosthesis.
\end{abstract}

Resumo. Este trabalho propõe uma solução para apoiar o processo de reabilitação de pacientes que sofreram amputação traumática ou cirúrgica de membros superiores. Considerando este cenário, espera-se fornecer uma ferramenta de treinamento que usa a Tecnologia de Realidade Aumentada (RA) para auxiliar os pacientes no processo de reabilitação. Espera-se que ao utilizar este recurso, os pacientes possam ser facilmente adaptados a uma prótese física.

\section{Introdução}

O estudo sobre a aplicação de novas tecnologias como Realidade Aumentada (RA) e Realidade Virtual (RV), aplicadas como instrumento de apoio ao processo de reabilitação física de pacientes que sofreram algum tipo de amputação, está em constante crescimento (KIRNER \& KIRNER, 2007). São reconhecidas diferentes técnicas que podem ser adotadas no tratamento destes indivíduos, dentre elas, a Eletromiografia (EMG) é uma das soluções de monitoramento que merece destaque, pois utiliza atividades elétricas constantes nas células musculares para realizar o monitoramento.

Especificamente, em relação à simulação de próteses para membros superiores, muitas aplicações são encontradas, as quais adotam hardwares ou softwares capazes de identificar e interpretar sinais mioelétricos, e a partir destes, constituir determinados movimentos. Este tipo de tratamento quando integrado a soluções de RA e RV, visam a recuperação de atividades musculares por meio de soluções com alto nível motivacional, uma vez que possibilita ao paciente identificar, visualmente, seus membros amputados. Segundo Trevisan et al. (2010), essa prática viabiliza estímulos cerebrais, necessários para a reabilitação do paciente. Dessa forma, o paciente pode visualizar a projeção do membro amputado, assim como todos os objetos do cenário real ao seu redor na tela do computador.

Reconhecendo o contexto apresentado, o objetivo deste projeto é a construção de uma solução computacional utilizando realidade aumentada e um nó sensor, que possibilite a simulação de um conjunto de atividades necessárias ao processo de reabilitação física de pacientes com amputações em membros superiores. Vislumbra-se também, que este estudo disponibilize um instrumento válido para fisioterapeutas acompanharem a evolução de seus pacientes. 


\section{Referencial Teórico}

De acordo com Abrahão et al. (2011), ao sofrer acidentes que resultem em amputações em membros do corpo, os indivíduos podem sofrer tanto de maneira física, quanto psicológica. E por isso, é relevante tentar restabelecer a liberdade e a mobilidade destes pacientes, resultando na melhor readaptação dos indivíduos a sociedade. Dessa forma, o estudo relacionado a próteses virtuais está crescendo nos últimos anos. Entretanto, a reabilitação é lenta e desgastante, o que acarreta em um alto grau de desistência dos indivíduos durante o período de tratamento.

Atualmente, as clínicas de reabilitação física utilizam várias técnicas para estimulação dos músculos de membros amputados. Dentre elas, a terapia do espelho, a qual consiste em uma técnica que utiliza um espelho interposto entre os membros superiores, é largamente utilizada. Esta técnica proporciona ao paciente a ilusão de visualização do seu braço amputado movimentando-se de maneira similar ao membro não afetado, induzindo a estímulos e favorecendo possíveis ganhos de mobilidade e coordenação nas áreas amputadas.

Desta forma, Soares (2003) destaca, a RA tem se apresentado como uma eficiente ferramenta em aplicações da área médica. Considerando o processo de reabilitação, a proposta da RA se torna uma ótima alternativa para realizar um tratamento mais atrativo e menos desgastante. Sendo assim, este trabalho tenta explorar através de uma solução computacional o uso de RA para o treinamento e adaptação, de pacientes com membros amputados que estimule o cérebro e os músculos durante as primeiras fases do processo de reabilitação. Logo após, a amputação, o coto não pode ficar exposto ao atrito e nem sofrer sobrecarga, dessa forma, próteses não podem ser utilizadas. Então, até que as próteses definitivas estejam aptas para a utilização, é possível realizar uma adaptação e estimulação das áreas do coto com RA. Nogueira et al. (2005), descreve que a utilização da RA pode proporcionar ao usuário uma maior imersão, combinando cenas virtuais e ambientes reais em um ambiente único.

\section{Metodologia}

Este projeto está sendo desenvolvido sob um método científico (CERVO et al. 2007), considerando para isto a experimentação, observação e coleta de dados relacionados a aplicação da RA como instrumento para o apoio ao processo de reabilitação física.

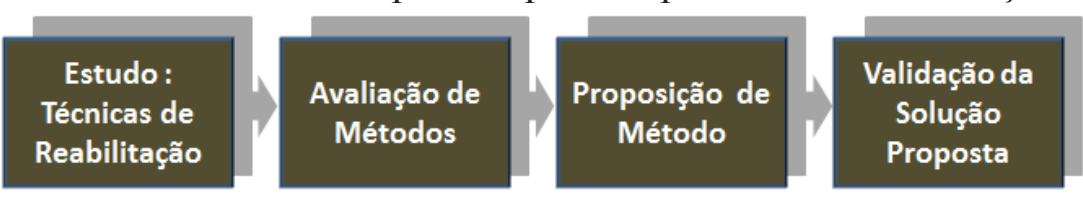

Figura 1. Organograma da metodologia.

Um conjunto de etapas, previamente definidas, sustentam as ações da pesquisa, conforme apresentado na Figura 01, as quais estão constituídas por: técnicas de reabilitação de pacientes com membros amputados, avaliação dos métodos estado da arte, propor um método para visualização da prótese e validação da solução proposta a qual foi desenvolvida, implementada e testada. Atualmente, este estudo encontra-se na fase de proposição de um método. Este estudo está sendo desenvolvido em uma parceria do Serviço de Reabilitação Física da cidade de Bagé/RS, com o apoio da Unimed Região da Campanha.

\section{Implementação}

Primeiramente, foi realizado um estudo sobre a área de reabilitação de membros amputados e RA, a partir disso, identificou-se que a solução mais pertinente seria a construção de um braço virtual, o qual possibilitaria um ambiente motivador aos 
pacientes amputados de membros superiores. A etapa seguinte, foi constituída da implementação e modelagem de um protótipo de braço virtual, o qual será utilizado para representar a prótese. Neste modelo, foi definido uma armadura de 21 ossos, o que permitiria a execução de movimentos mais reais da prótese, possibilitando assim, uma sensação mais realista ao paciente. Após a modelagem do protótipo foi utilizado o software Blender ${ }^{1}$ para desenvolver as movimentações do protótipo. A figura 2 apresenta a modelagem do protótipo de braço, assim como na figura 3 são apresentados os movimentos básicos a serem realizados. Estes movimentos são: fechamento da mão, flexão dos dedos: polegar, indicador, médio, anelar e mindinho.

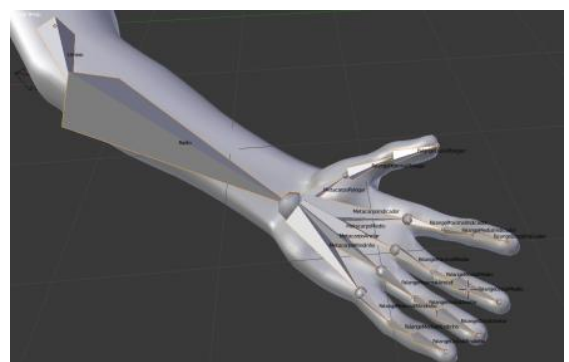

Figura 2. Construção do braço virtual.

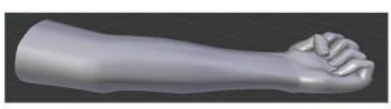

(a) Preensâo da mâo.

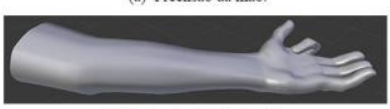

(c) Flexão do dedo indicador:

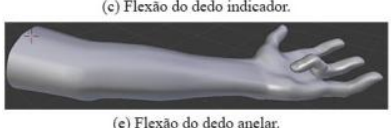

Figura 3. Seis movimentos básico da prótese virtual.

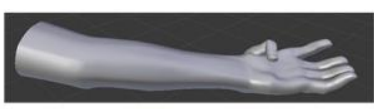

(b) Flexão do dedo polegar.
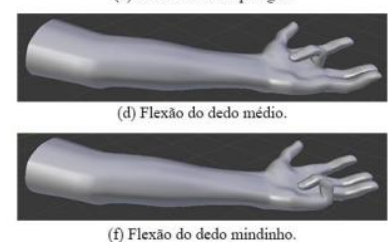

A interação do paciente com o ambiente virtual de treinamento é efetuada através da tecnologia Unity ${ }^{2}$. O processo de movimento da prótese virtual é definido por 3 etapas, são elas: aquisição, processamento e resposta. Assim, estas etapas são responsáveis pela aquisição dos comandos do teclado, e logo após, pela determinação do movimento através de comandos do ambiente no Unity. Desta forma, é efetuada a movimentação da prótese de acordo com a determinação do movimento obtido no módulo de processamento. A figura 4 representa um organograma com a representação do conjunto de etapas do movimento da prótese virtual.

Após construção do membro, a prótese foi associada a um marcador. Portanto, quando a câmera localizar o marcador o objeto associado a ele é inserido na tela em tempo real, como apresenta a figura 5.
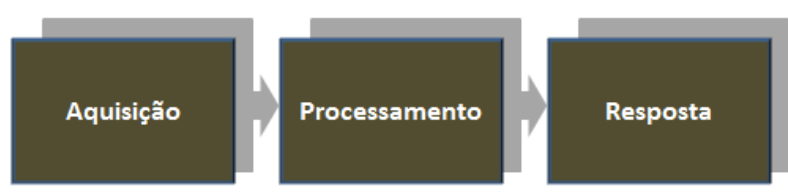

Figura 4. Organograma das etapas de movimentação da prótese.

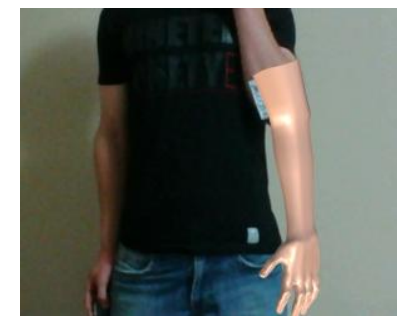

Figura 5. Prótese virtual projetada sobre o marcador.

\section{Conclusões Parciais}

Neste trabalho é proposta uma solução computacional que permite a utilização de Realidade Aumentada na área de saúde, mais especificamente, no tratamento de pacientes com membros amputados. A proposta é baseada nos benefícios da técnica do

\footnotetext{
1 Project Blender - https://www.blender.org/ - programa que permite a geração de modelos tridimensionais, apresentações, aplicações interativas, animações.

2 Motor de Jogo 3D Unity - https://unity3d.com - aplicação para o desenvolvimento integrado que fornece uma funcionalidade pioneira para criação de jogos e outros conteúdos interativos.
} 
espelho, e visa controlar os movimentos de um braço virtual através de sinais mioelétricos. Este ambiente simula movimentos virtuais mediante a uma máquina com interface gráfica, onde através da interatividade e imersão do paciente, espera-se estimular áreas motoras do córtex e de feedback visual. Até o momento, foram efetuadas as implementações iniciais do ambiente de RA, as quais apresentaram resultados satisfatórios na determinação e execução dos movimentos. Como próximos passos, pretende-se utilizar um microcontrolador conectado a sensores de sinais mioelétricos para a coordenação do protótipo de braço em RA, além de adicionar algumas ações ao protótipo, tais como, segurar um objeto e movê-lo de lugar.

Considerando que a técnica do espelho é uma das técnicas mais efetivas, pretende-se, futuramente, criar uma série de treinamentos e níveis para os diferentes tipos de pacientes. Assim, espera-se melhorar os resultados dos tratamentos antes da prótese real, visto que, as técnicas convencionais nem sempre geram resultado. Em suma, este projeto visa implementar uma ferramenta que auxilie fisioterapeutas no processo de tratamento dos pacientes de membros amputados superiores, colaborando para uma melhor qualidade de vida das pessoas e agilizando o processo de reabilitação.

\section{Referencias}

ABRAHÃO, L. C. L; LAMOUNIER E. A.; Realidade aumentada on-line aplicada à simulação de um membro superior. Aplicações Médicas. Uberlândia-MG: FAPEMIG. 2011.

CERVO, A., BERVIAN, P., DA SILVA, R. "Metodologia Cientifica”. 6a ed. 2007.

KIRNER, C.; KIRNER, T.G.; Virtual Reality and Augmented Reality Applied to Simulation Visualization. In: El Sheikh, A.A.R.; Al Ajeeli, A.; Abu-Taieh, E.M.O. (Org.). Simulation and Modeling: Current Technologies and Applications. 1 ed. HersheyNY: IGI Publishing, 2007, v. 1,p. 391-419.

MATTIOLI, F. E. R.; CAETANO, D. S. D.; GOMES, W.;LAMOUNIER, E. A.; CARDOSO, A. Utilização de redes neurais para a classificação de sinais EMG aplicados no controle de próteses virtuais de mão. [Anais Do] WRVA'20107 ,WorkshopDeRealidade Virtual e Aumentada, SãoPaulo: SBC, 2010.

NOGUEIRA, K. L.; CARDOSO, A.; LAMOUNIER, E.; Uma aplicação de realidade aumentada no auxílio da adaptação de próteses em membros superiores. WARV. Uberlândia, 2005.

RIBEIRO, M.; ZORZAL, E. Livro do pré-simpósio, XIII Symposiumon Virtual and Augmented Reality. Uberlândia-MG: SBC, 2011. 150 p.

S. HERLE, P. RAICA, G. LAZEA, R. R., M. C., AND L. TAMAS. Classification of surface electromyography signals for control of upper limb virtual prosthesis using time- domain features. Proceedings of the 2008 IEEE International Conference on Automation, Quality and Testing, Robotics, pages160-165, 2008.

SOARES, A.; ANDRADE, A.; LAMOUNIER JÚNIOR, E. A; and CARRIJO, R. The development of a virtual myoelectric prosthesis controlled by an EMG pattern recognition system based on neural networks. Journal of intelligent information systems, 2003.

TREVISAN, C.M., TRINTINAGLIA, V.; Efeito das terapias associadas de imagem motora e de movimento induzido por restrição na hemiparesia crônica: estudo de caso. Fisioterapia e Pesquisa, São Paulo, v.17, n.3, p.264-9, jul/set. 2010.

ORTIZ-CATALAN M, GUĐMUNDSDÓTTIR RA, KRISTOFFERSEN MB, et al.; Biosignal Processing and Computational Methods to Enhance Sensory Motor Neuroprosthetics, 2016. 